\title{
Zur Behandlung Kriegsverletzter mit mediko-mechanischen Behelfsapparaten.
}

\author{
Ton \\ Sanitätsrat Dr. Friedeberg-Nagdeburg, \\ \%. \%t. Oberstabsint, bommandiert zum Sunititsumt des [V. A. K. \\ Hit lo Abbildungen in Text. \\ (Eingegtingen am 21 . Marz 1919.)
}

Am Ende des Kriegjahros 1915 erwies sich die Einrichtung einer nedikomechanischen Abteilung fïr Leichtverwundete und Kranke im Eiappenbereich der IV. Arnee als dringende Notwendigkeit. Bis dahin wiaren fast alle dorartig behandlungsbedurftigen Wamschaften und offiziere, abgeseben vou geringfügigen Leiden, den Fachlazaretten dor Heimat zngetührt worden, da im Etappengebict Einrichtungen dieser Art nur in versehwindendem Lnfang bestanden. Die UnregelmäBiglieit der Bahnverbindungen und der zeitraubende Hin- und Richtranswort einersoits, die l'berlastung der heimatlichen Lazarette und Fachïrzte anderseits lieben es wïnschenswert erscheinen, möghichst bald in einem geeigneten Etappenlazarett pasiende Einrichtungen zu treffen. Die Leichtkrankenabteihns im Etappenhauptort Gent, die zusammen mit der Genesungsabteilung ïber annähernd 1600 Betten verfügte und an welche eine Handwerkerabteilung angeschlossen war, entsprach in jeder Weise den geplanten Zweeken. Dic großen, luftigen Hallen und gerïnmigen Zimmer stammten von der Genter Weltausstellung und waren in äußerst praktischer Weise schon im Herbst 1914 zum Lazarett hercrerichtet und später erheblich erweitcrt worden. Die freie Lage in parkartiger Lomgebung und geniigender Platz für Gymnastik und Turrübungen waren ein weiterer Vorteil dieses Lazaretts.

Da schnelles Handeln greboten war und mit dem einfachsten Material gerechnet werden mul3te, auch geschulte Hechaniker nicht vorhanden waren, konnte ich mit Hilfe von einigen gewandten Schlossern, Tischlem und Sattlern, die aus dem Bestand der Kranken entnommen wurden, lediglich Apparate ats Eisen und Holz herstellen; Blei und Leder standen num in versehwindender Menge zur Verfïgung. Mischinen mit elektrischem oder anderem Motorbetrieb kanen für uns nicht in Frage. Als mechanische Medien dienten Rollen, Pendel, Gewichte und Eisenfedern. Es galt einerseits möglichst solche Apparate zu scbaffen, die lejcht zu handhaben waren, anderseits solche zu vermeiden, welche durch den Gebrauch leicht beschädigt und verhälnismäßßig schnell abgenutzt 
werden konnten. Diese letzte Bedingung erwies sich später als durchaus be rechtigt, da schon nach wenigen Wochen unser durchschnittlicher Krankenbestand mehr als 80 betrug.

An die medikomechanische Station war eine solche für Massage angegliedert. Als wertvolle Unterstiitzung der Behandlung dienten ferner die in Lazarett vorhandenen Einrichtungen für Bäder-, Heibluft-, Freiluft- und Filektrotherapje. Als diagnostisches Hilf smittel stand eine gatgeleitete Rontgenabteilung zur Verfügung. Weiter wurden ein orthopädischer Turnkursus sowie Turnspiele eingerichtet, an denen sich die Genesenden mit Eifer und Erfolg beteiligten.

- Um die Apparate möglichst auszunutzen und gleichzeitig Úbermüdung der Kranken zu vermeiden, übte die Mehrzahl der Patienten nur kïrzere Zeit, dafür jedoch zweimal täglich. Es veranlaßte dies keine Schwierigkeit, daz die Kranken mit wenigen Ausnahmen im Lazarett selbst untergebracht waren.

Das Material unserer Fille umfaßte in erster Linie Schußverletzte, jedoch waren auch Nachbehundungen ron Stichverletzungen, Verbrennungen und Operationsuarben zahlreich. Winen beträchtlichen Prozentsatz bildeten ferner Fälle ron Versehüt,ung, sei es, daß Quetsehungen des Bewegungsapparates oder mehr nervöse Störungen hierbei in den Vorder grund traten. Weiter boten Knochenbrüche, besonders der Fußknöchel, sowie Verrenkungen, namentlich Schulterluxationen, Anlaß zur Behandlung, um hier nur der wichtigeren Verletzungen zu gedenken. Von inneren Leiden wurden hauptsachlich Prheumatismus, seltener Gicht und Ischias behaudelt. Bei jearotikerm leichteren Grades warde mediliomechanische Behandlung als suggestive Therapie wiederholt mit Erfolg ansgefüht, wobei die Verlegung dieser Paticnten in unser Lazarett, also in ein anderes Milieu, vielleicht fordernd wirkte. Später wurden diese Fille, als Speziallazarette hierfür eingerichtet wurden, unmittelbar dorthin übersandt.

Anfangs wurden unserer Station eine reichliche Zahl von fällen äßerwiesen, die wir von vornherein zurückweisen mußten, da sie für uns nicht geeignet waren. Es waren dies nimentlich Verletzte mit kaum verheilten, Frischen Narben; ferner solche mit der Diagnose, "Verschüttung", bei denen sich Wirbelverletzungen erwiesen; schließlich Patienten, bei denen bereits völlige Versteifung und erhebliche Lähmung eingetreten war. Später lamen diese Fehler in der Beurteilung nur noch vereinzelt vor, dank günstigen Erfolges unserer Fortbildungstiurse für jüngere Arzte. Während es leicht war, alle diese Fialle sofort von der Behandlung auszuschließen. machte es nicht selten anfangs Schwerigkeit, schon wor oder bei Beginn der Behandlung zu bestimmen, wie lange Zeit dieselbe voraussichtlich in Anspruch nehmen wïrde. Es bedurfte für uns erst einiger Ubung, um in diesem prognostischen Urteil eine gewisse Sicherheit zu erlangen. Dies war insofern wichtig, als grundsätzlich diejenigen Patienten in die Heimat geschicht werden sollten, bei denen längstens im Laufe eines Monats keine Heilung oder keine derartige Besserung zu enwarten war, daß sie kriegsverwendungs-bzw. garnisonverwendungsfähig für Etappe wurden. Allnïhlich wurden wir jedoch so geschult, daß es später verhältnismäligig selten vorkam, daß Kranke erst nach vergeblicher wochenlanger Behandlung Feimatlazaretten ïberwiesen werden muBten. 
In folgendem beschränke ich mich darauf, von unseren insgesamt 30 Apparaten bier nur diejenigen zu erörtern, wolche mehr oder weniger von bekaunten Modellen abweichende Konstruktionen hatten; von Einzelheiten glaube ich um so mehr absehen zu können, als Abbildungen mit MaßBangabe und kurzen Erläuterungen beigefügt sind. Mehrere Apparate waren in cinem Modell zu verschiedener Handhabung vereinigt, um Platz und Material zu sparen.

Zur Behebung von Steifigkeit und Verkrümmungen der Fingergelenke diente ein sogenanter Tastendruckapparat (Abb. 1). Er besteht aus einem Holzkasten, auf welchem an einer Metallachse beweglich sich fünf Holztasten befinden, für jeden Finger eine. Dex hiirzere Hebel dient zum Aufsetzen der Finger, der längere zur Befestigung kleiner Wetallgewichte mittels fünf Häkchen an der Untersejte desselben. Der Abstand der einzelnen Häkchen von einander beträgt $5 \mathrm{~cm}$. Das Metallgewicht ist $300 \mathrm{~g}$ schwer, ein Maß, das sich nach meinem Dafürhalten als das geeignetste erwies. Je weiter das Gewicht sich dem Ende des längeren Hebelarmes nähert, je größere Kraft ist naturgemäß nötig, um den kürzeren Hebelarm herabzuditichen. Durch Ubung mit diesem Apparat. kann man häufig bei frischen Fälen in ziemlich kurzer Zeit eine Stärkung der

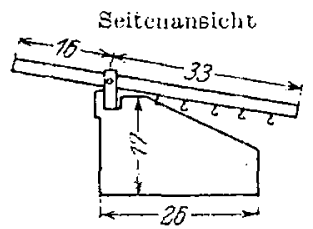

Abb. 1. (Holz, Metallachse und Gewichte)

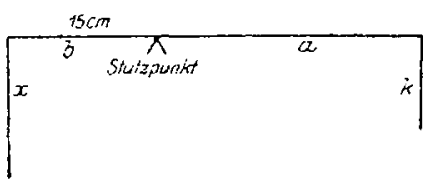

Abb. 2.

Fingerflexoren durch das Herabdrücken der Tasten erreichen, währeud beim Aufhören des Druckes automatisch eine Dehnung der Extensoren, allerdings in geringerem Maße, erzielt wird. Am besten vergleicht man die. Wirkung mit der durch Klavierspielen erreichten. Der Vorteil unseres Apparates besteht jedoch darin, dab die trbeitsleistung variabler jst, und dab man sie mathematisch genau berechnen kinn. Es geschieht dies nach der Formel $\times$. h = a. k oder $\mathrm{x}=\frac{\mathrm{a} \cdot \mathrm{k}}{\mathrm{b}}$, wobei b fraftalm $(15 \mathrm{~cm}$ lang $)$, a Lastam, k Last und $x$ Kraft bedeutet (siehe Abb. 2). Last h betrïgt insgesamt $525 \mathrm{~g}$. Da nun, wie gesagt, der Lastam durch rerschiedenes Anhä̈ngen der Gewichte variiert (zwischen 10 und $30 \mathrm{~cm}$ ), ist es möglich, für jede Stellung des Ciewichtes $x$ (Kratt) zu becechnen, und zwar betrïgt

$$
\begin{aligned}
& \mathrm{x}_{1}=\frac{525 \cdot 10}{15}=350 \mathrm{~g} \\
& \mathrm{x}_{2}=\frac{525 \cdot 15}{15}=525 \mathrm{~g}
\end{aligned}
$$

usw. jedesmal $175 \mathrm{~g}$ mehr bis $x_{5}=1050 \mathrm{~g}$.

Ein weiterer Fingerbeuge- und Streckapparat wurde in Cestalt eines Gewehrkolben konstruiert, an dessen Unterseite sich ein gekrïmmter Haken zum Abziehen für don Finger befindet. Lin den Apparat wieder nach 
Zur Behandlung Kriegsverletzter mit mediko-mechanischen Beheifsapparaten. 127

dem Abriehen in Stellung zu bringen, ist eine Kurbel angebracht. Nähere Erläuterung bringt Abb. 3. Für die spätere Handhabung des Gewehres beim Schießen ist diese Vorïbung oft nützlich gewesen.

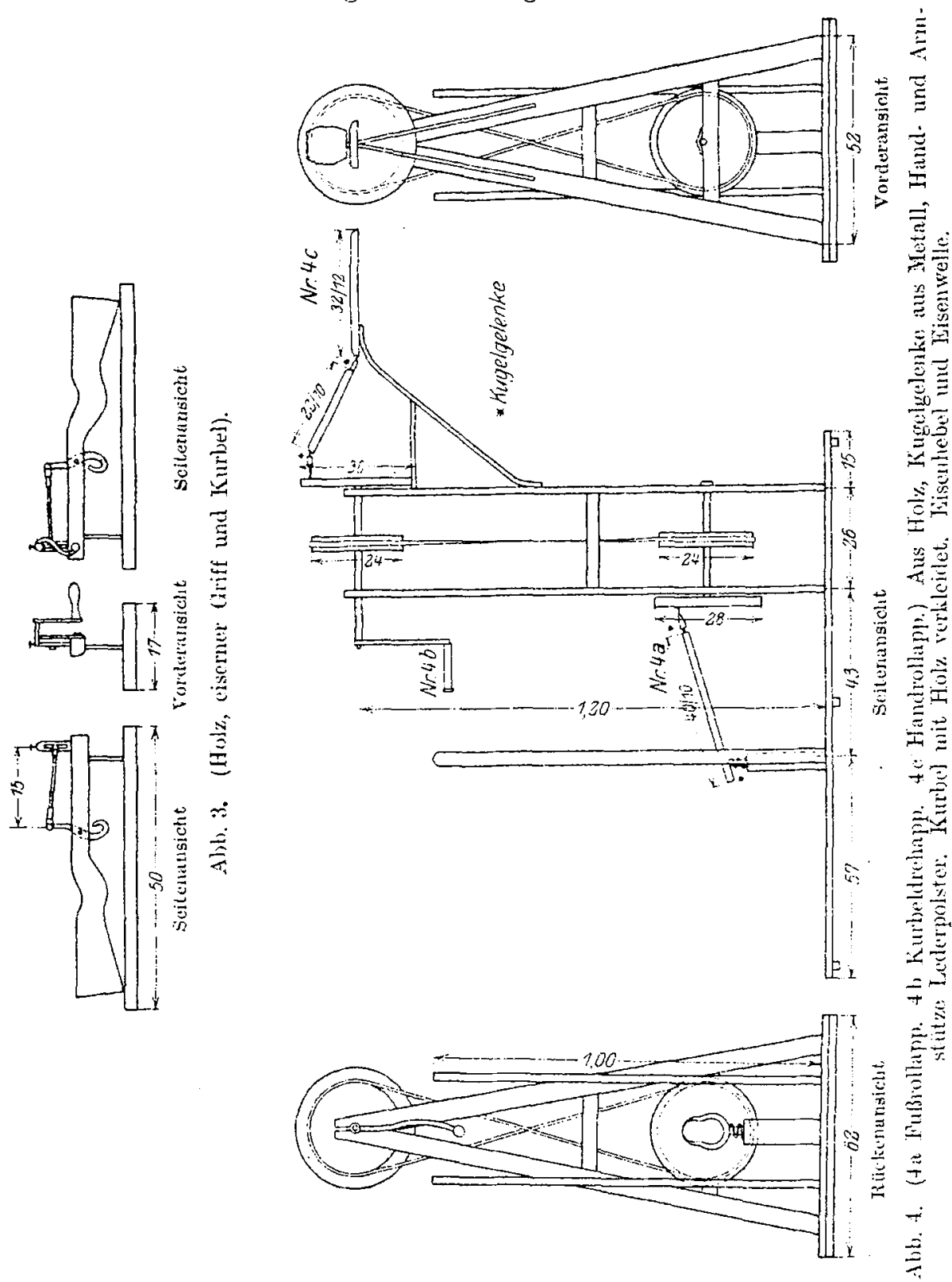

Zur Behandlung vou $H$ andgelenksversteifung dient Apparat te, durch den passiv Rollen des Handgelenks erzielt wird, Der Bewegungsradius lii.jt sich durch Schraubenverstellung in mäbigem Lmfang erweitern. Zum Antrieb 
dient eine Kurbel (Abb.4b), die von einem zweiten Patienten gehandhabt werden muß, der hicrdurch Drehung im Ellbogen-. und Schultergelenk ïben

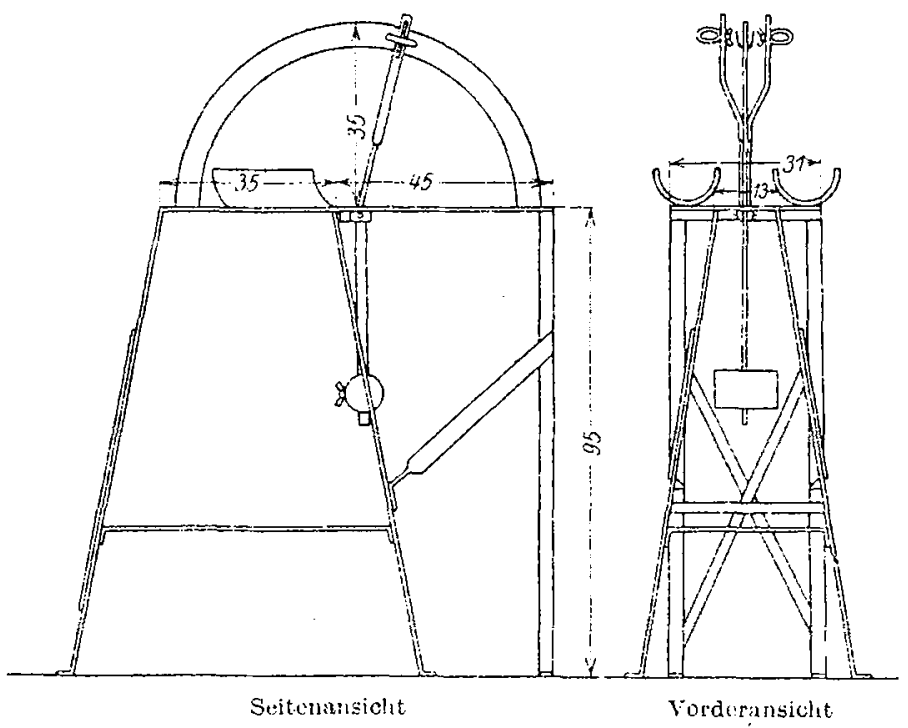

Abb. 5. (Konstruktion aus Eisen, Armstützen aus Leder).

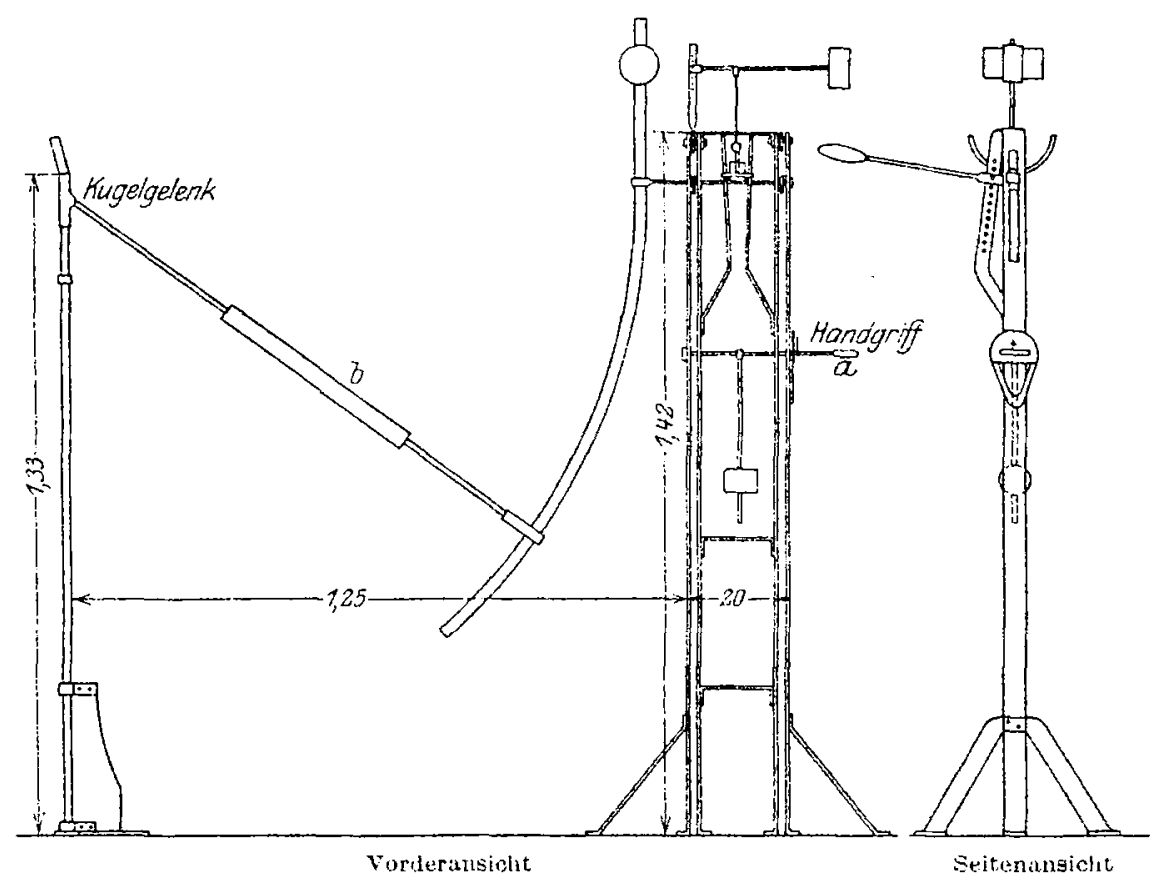

Abb. 6. (Konstmktion aus Eisen nit Kugelgelenken).

kunn. Durch denselben Kurbelantrieb wird gleichzeitig ein Fußrollapparat bedient (Abb. $4 a)$. 
Einen Apparat zum Strecken und Beugen des Unierarmes stellt Abb. 5 dar. Der Kranke sitzt auf einem Stuhl an der linken bzw. rechten seite des Apparates, je nachdem er mit dem linken oder rechten Unterarm iibeu soll. Der Oberarm wird auf eine gepolsterte Armstiitze ilufgelegt oder festgeschnallt, wïhrend mit dem Unterarm durch Druck der Hand auf einen Griff eine aktive Bewegung imerhalb eines Halbkreises nach oben (Beugen) oder nach unten (Strecken) ansgeïbt wird. Auf der Peripherie des Halbhreises sind im Abstand von je 5 cm Zahken aufgemalt, so daß Fortschritte der Beweglichkeit unmittelbar festgestellt werden können. Das zur Hemmung dienende

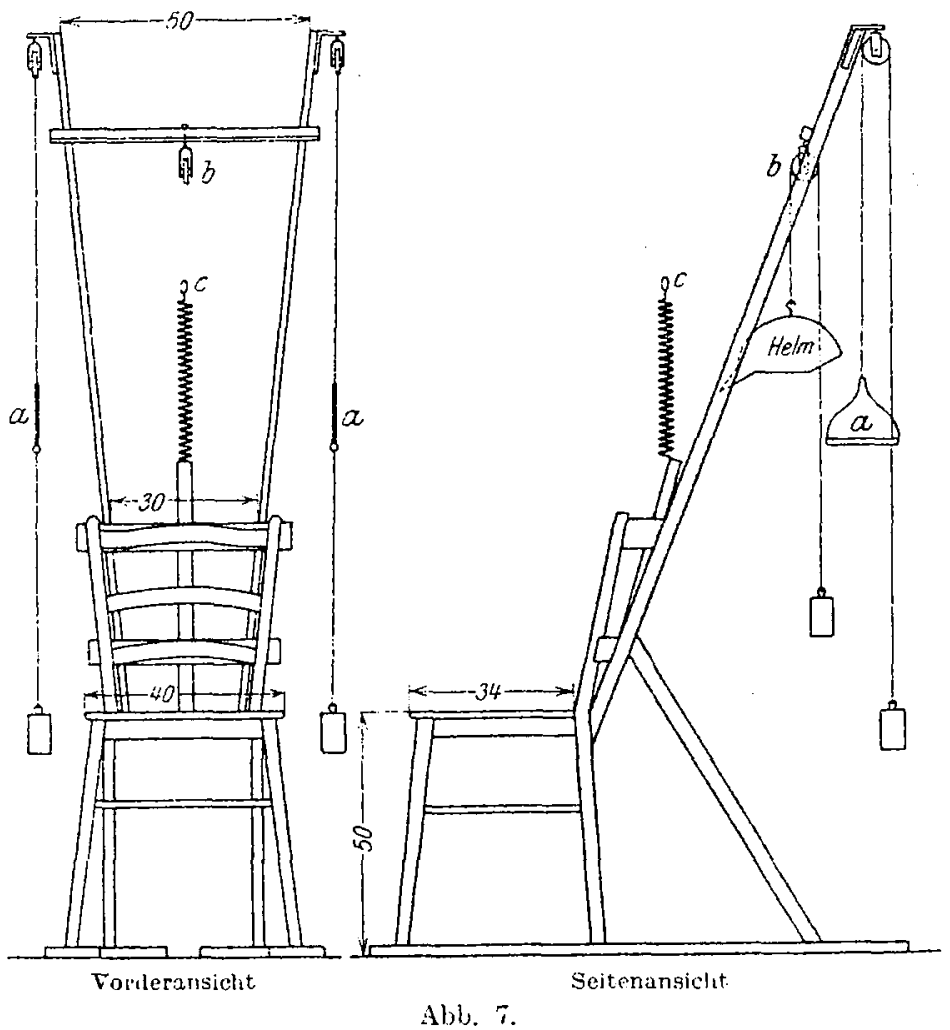

Metallgewicht ist auf seiner Achse verschieblich, wodurch die Kraftleistung des Ubenden nach Bedarf vermehrt oder vermindert werden kann.

Der folgende Apparat ist auf der einen Seite für ahtive Pro- und Supination des Unterarmes bestimmt (Abb. 6a), auf der anderen dient ex zum Kreisen des Oberarmes im Schultergelent (Abb, 6b). Das Stativ ist verstellbar, kann somit der Größe des Patienten angepaßt werden, auch kann ferner durch Verschieben des Handgriffes auf seinem Achsenbogen ein verschiedener Umfang des Armkreisens ermöglicht werden.

Gleichfalls zum Armrollen im Schultergelenk, jedoch in geringerem Umfang als der vorige Apparat und mit dem Unterschied, daß der Kranke sitzend übt, ist Apparat 7 a bestimmt. Verbunden hiermit ist eine Vorrichtung 
zum Beugen und sitrecken des Kopfes (Abb. Th). Zur Kopflefestigung dient ein Helm, dessen unterer Pand abgeschrïgt ist und welcher unter dem

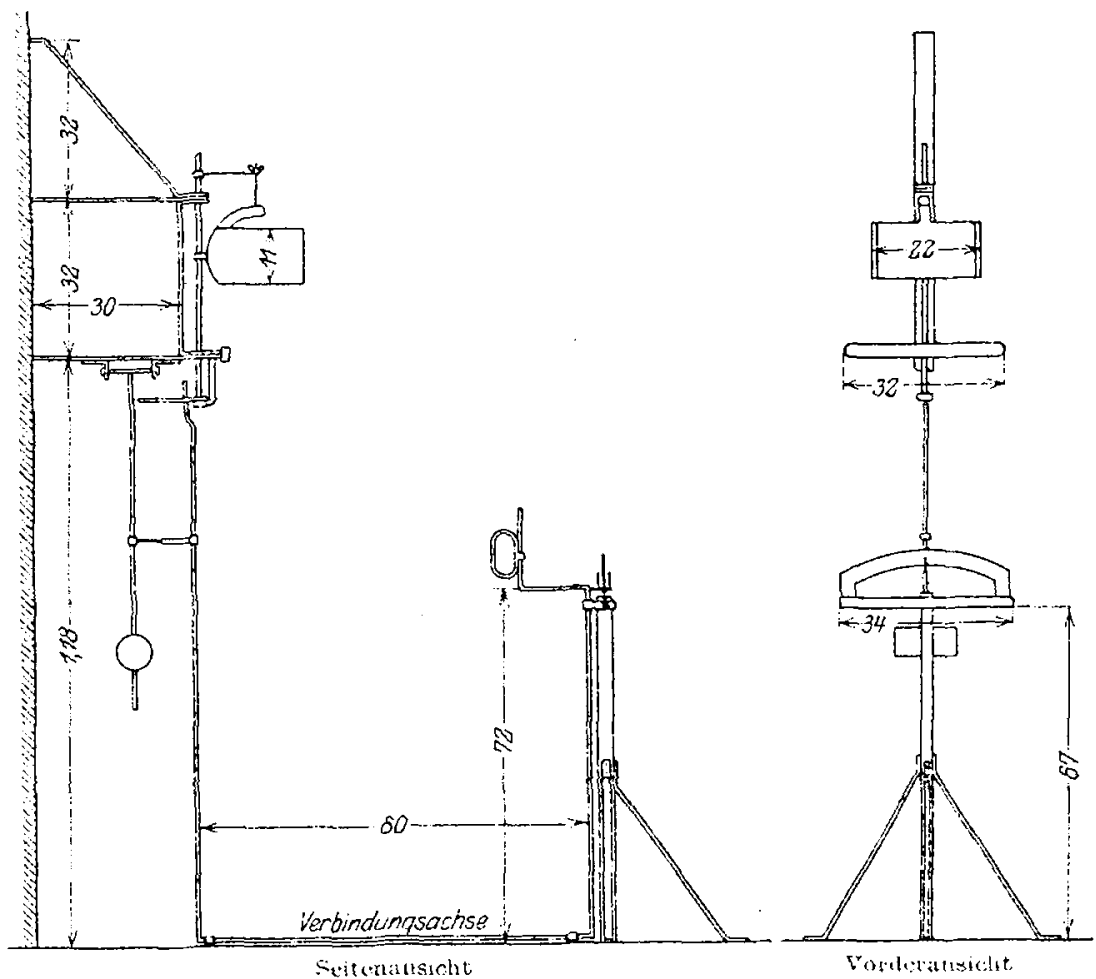

Abb. 8. (Konstruktion in Eisen. Koptstiatz: uit Filzpolsterunge).

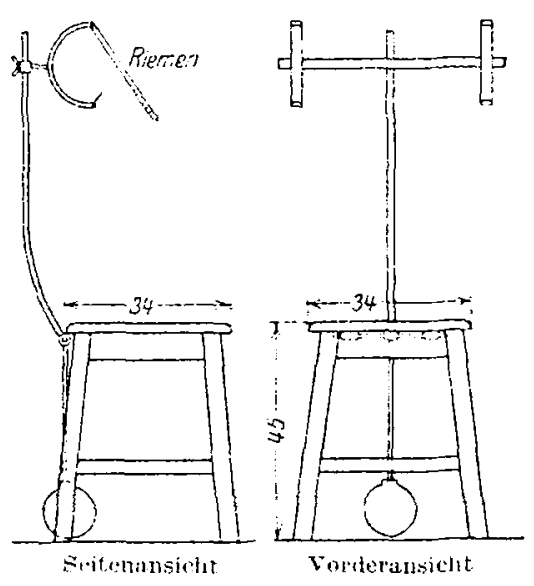

Abb. 9.

Kimn festgeschnallt wird. Zum aktiven Kopfrollen kinn die greiche Vorrichtung mit Hilfe einer binenfeder lonutyt werdon (Ab), $;$ (c). Nimentlich zur Dehrnung von Nackennarben nach SchuBverletzungen und Furunkeloperationen hat sich diese thung bewilhrt.

the dringendes Bedirrfnis erwies sich ferner ein Apparalt, mit welchem passives Seitwi rtsdrehen des Kopfes bewirkt werden konute. Die Konstruktion veranlakte erhebliche schwierigkeit und gelang schliephich daumel, daf ein Verbindungsachse zwischen dem 'Trïger der Kopfstïtze und einem Handgriffe hergestellt worde. Der sitzende Kranke kiann durch Verschicken des Handgriffes nach rechts den Kopf mach links drehen und umgekehrt (AWh). S). 
Wahrend der Bau eines Apparates zum Vorwäts- und Aufwartsbeugen des Rumpfes ziemlich einfach war, und seine Einrichtung aus Abb. 9 leicht ersichtlich, kostete die Konstruktion eines solchen für aktives seitliches Pumpfbengen viel Zeit and Wïhe, da die richtige Zentrierung erst nach längeren Versuchen gefunden wurde (Abb. 10). Jje Lösung des Problems gelang dadurch, dab für das obere Stiick des beweglichen Teils die Form eines Holzkrenzes gew ählt wurde, an dessen seitlichen Enden Griffe für den Patienten zum Festhalten mit ansgestreckten Armen angebracht warden. Freilich geniigt dieser Apparat nur für Kranke ohne erhebliche Rumpf- und Hüftversteifung; schwerere Fille dieser Art eigneten sich $j \mathrm{j}$ an sich nicht fïr misere Bebandlung.

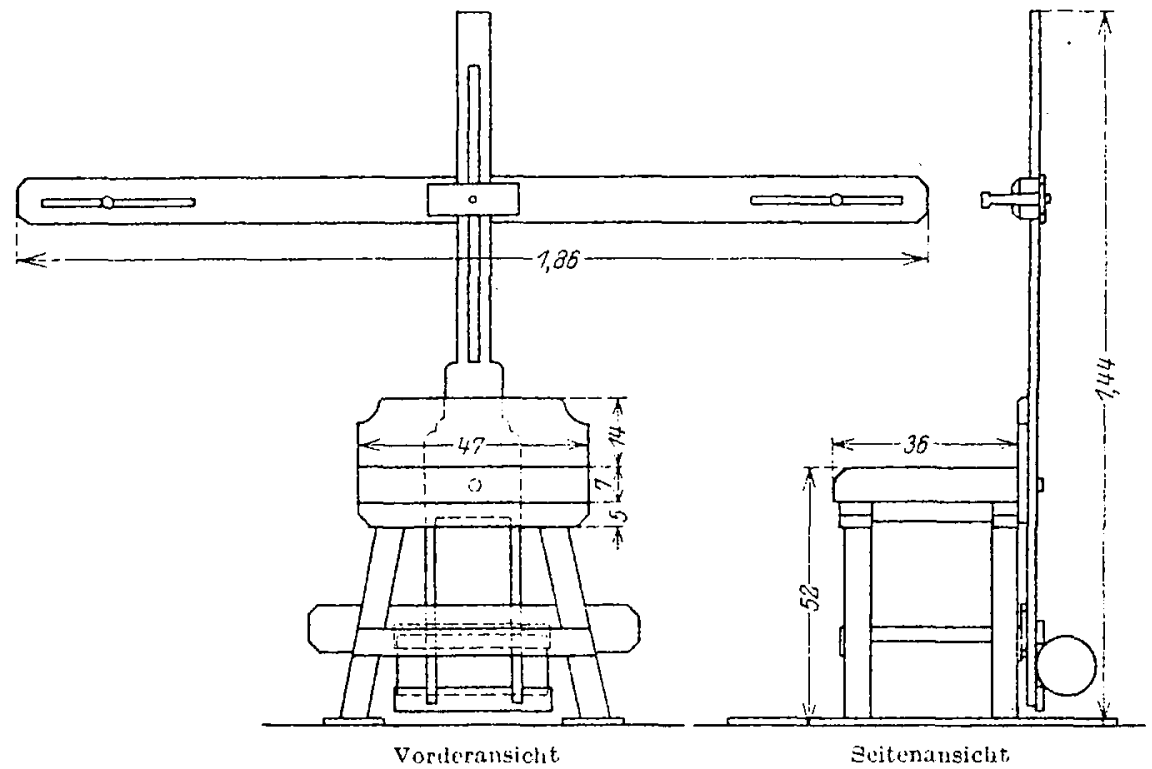

Abb. 10. (Holz mit Metallgewirhten).

Hiemit stände ich am Sichluß meiner Betrachtungen. Die Veröffentlichung dieser Zeilen konnte ans üuBeren Grïnden erst jetzt nach Beendigung des Krieges erfolgen. Ein Himweis zur Herctellung einfacher Behelfsmittel dïrfte jedoch vicht unangebracht sein in einer Zeit, wo noch zahlreiche Kranke, die medikomechanischer Behandhng bedürfen, in den Lazaretter weilen, und wo die vorhandenen Apparate kalum genigen, um den Bedarf zu decken. Hierau kommt, daß Neuauschaffung und Ersatz fabrikmäßig hergestellter, kunstvoller Apparate für den Orthopiaden vorerst auBerordentlich sehwierig und ungehewer kowtspielig sein wird. Daher ist vielleicht manchem beschäftigten Kollegen, der nicht genügend Zeit zu mechanischen Versuchen hat, diese oder jene meiner Anregungen nicht unwilkommen. 